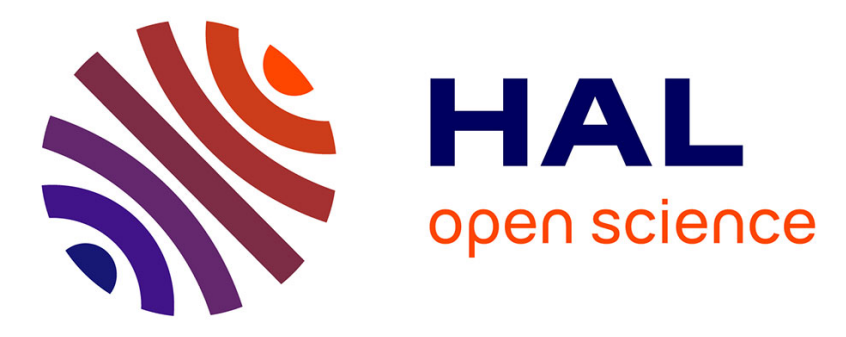

\title{
Les implications philosophiques de la théorie de l'action collective
}

\author{
Armand Hatchuel
}

\section{To cite this version:}

Armand Hatchuel. Les implications philosophiques de la théorie de l'action collective: Commentaire à l'article de Baptiste Rappin: "Le glas des métaphysiques de l'action a-t-il sonné? Heidegger Lecteur de Hatchuel ". Revue internationale de psychosociologie et de gestion des comportements organisationnels, 2017, 1 (XXIII ), pp.187-191. hal-01502891

\section{HAL Id: hal-01502891 \\ https: / hal-mines-paristech.archives-ouvertes.fr/hal-01502891}

Submitted on 9 Jun 2017

HAL is a multi-disciplinary open access archive for the deposit and dissemination of scientific research documents, whether they are published or not. The documents may come from teaching and research institutions in France or abroad, or from public or private research centers.
L'archive ouverte pluridisciplinaire HAL, est destinée au dépôt et à la diffusion de documents scientifiques de niveau recherche, publiés ou non, émanant des établissements d'enseignement et de recherche français ou étrangers, des laboratoires publics ou privés. 


\section{Les implications philosophiques de la théorie de l'action collective.}

Commentaire à l'article de Baptiste Rappin: "Le glas des métaphysiques de l'action a-t-il sonné ? Heidegger Lecteur de Hatchuel».

Revue internationale de psychosociologie et de gestion des comportements organisationnels, ESKA, 2017, 1 (XXIII ), pp.187-191. <hal-01502891>

Armand Hatchuel

MinesParisTech /PSL Research University CGS-I3 UMR 9217.

Baptiste Rappin a eu la courtoisie de m'inviter à commenter son article consacré à un examen de la «théorie de l'action collective », connue aussi sous le nom d'« axiomatique savoirs-relations ${ }^{1}$. Cette théorie - que je développe, avec quelques collègues- introduit la notion de «métaphysique de l'action » pour caractériser un point aveugle dans la construction des sciences sociales et pour élaborer une théorie de l'action collective mieux fondée. A ce titre, la théorie de l'action collective mériterait, selon l'auteur, une attention particulière. Son examen se justifierait, de plus, par la place importante que cette théorie occuperait dans les sciences de gestion contemporaines.

Cependant, l'analyse proposée dans cet article reste sans lien avec les sciences de gestion et ne s'intéresse pas aux problèmes des sciences économiques et sociales qui sont à l'origine de la théorie de l'action collective. Baptiste Rappin retient surtout que cette théorie exprime une ambition philosophique universelle qui rejoindrait les tentatives actuelles de «sortie de la métaphysique ». Tentative, qui réaliserait un véritable «tour de force», mais qui resterait néanmoins vaine parce que prisonnière de présupposés idéologiques que l'auteur se propose d'abord de dévoiler puis ensuite de condamner en prenant le parti - pour le moins extravagant, compte tenu du champ de la théorie- d'invoquer Heidegger à cette fin.

L'article s'organise autour de trois critiques. La première affirme que la théorie de l'action collective reste in fine une métaphysique. La deuxième critique soutient qu'elle relève de l'idéologie cybernétique, que l'auteur considère, en suivant Heidegger, comme la matrice dominante de la pensée occidentale, et celle qui a préparé une technicisation du monde. La troisième critique enfin, parachève le verdict en rappelant la condamnation par Heidegger de

\footnotetext{
${ }^{1}$ Ou encore « Axiomatique S/R».
} 
«l'arraisonnement» du monde par la technique, cette dernière étant conçue comme un schème général et aliénant de la civilisation présente. En résumé, la « théorie de l'action collective », qui veut refonder les sciences de gestion, serait un avatar sophistiqué et subtil - mais un avatar quand même- de la pensée technicienne. Elle tomberait donc sous le coup de l'anathème Heideggérien qui, semble-t-il, reste, pour Rappin, sans appel.

L'article surprend d'emblée par le contraste manifeste entre deux postures intellectuelles de l'auteur. D'abord, celle d'un lecteur curieux, attentif et sensible à une certaine finesse de la théorie de l'action collective. Mais vient ensuite, une démarche critique sommaire, peu soucieuse de convaincre et surtout pressée d'exprimer un credo préétabli. Car, l'auteur assène, sans précautions, la thèse - parmi les plus connues de Heidegger - que la pensée occidentale, y compris la science, serait prisonnière du schème technique général. En découle que la théorie de l'action collective, qui se veut simultanément science de l'action sociale et science de l'action technique, tombe naturellement sous le coup de cette condamnation générale. On comprend vite que le sous-titre de l'article paradoxalement flatteur à mon égard - "Heidegger lecteur de Hatchuel » - annonçait plutôt les foudres du Maître penseur et qu'il ne s'agissait plus d'un débat académique.

Quel peut donc être l'objet de ce commentaire? Les lignes qui suivent seront surtout consacrées à des précisions qui montrent que les deux premières critiques de Baptiste Rappin reposent sur des interprétations erronées de la théorie de l'action collective. Quant au recours à Heidegger, il serait grotesque de s'arrêter aux engouements philosophiques de l'auteur en engageant ici, à la va-vite, une discussion sur l'un des philosophes les plus controversés du 20è siècle. Je crois beaucoup plus utile d'indiquer aux lecteurs intéressés quelques implications philosophiques de la théorie de l'action collective qui ont un intérêt pour les problèmes contemporains.

Revenons d'abord aux deux premières critiques.

1. La théorie de l'action collective n'a jamais eu pour but de «sortir de la métaphysique ». Un tel énoncé, trop vague, n'a aucune signification scientifique et opératoire. Elle s'est attachée - comme le rappelle le titre de l'article- à bien délimiter, dans un corpus déterminé, une catégorie particulière de propositions, celle des «métaphysiques de l'action ». Il s'agit d'un ensemble de principes explicatifs de l'action collective humaine qui ont pour conséquences : i) de faire conceptuellement disparaitre l'action collective elle-même; ii) de n'avoir aucune valeur empirique. Ces métaphysiques de l'action sont faciles à décrire puisqu'elles réduisent l'action collective soit à l'intervention d'un sujet unique 
soit à la réalisation d'un principe unique, qui, dans chaque cas, «totalisent » l'action (son déroulement, son histoire,...). Chacun peut vérifier que dans la formation des sciences économiques, sociales et humaines, ainsi que dans celle des théories politiques, le recours à de telles «métaphysiques de l'action » a été constant: le peuple, le roi, le prolétariat, l'Etat sont des exemples bien connus de sujets totalisateurs. Par ailleurs, le profit, le progrès des connaissances et des techniques, la démocratie ou l'équilibre de marché constituent des principes totalisateurs.

Il faut remarquer que la réduction de l'action collective à un sujet ou à un principe totalisateur ne tient pas seulement à des observations insuffisantes. On y est d'autant plus enclin qu'il y a de grandes difficultés conceptuelles à penser la transformation conjointe des sujets (toujours multiples) et des principes d'action (toujours multiples). Or, c'est précisément, cette coévolution, créatrice ou destructrice, dont plusieurs chercheurs, au sein des sciences de gestion contemporaines, veulent rendre compte.

Ce dualisme (sujets/principes, savoirs/relations) est le plus souvent perçu comme un obstacle conceptuel qui impose de rabattre la pensée, soit sur les sujets, soit sur les principes. Pour la théorie de l'action collective, il s'agit au contraire d'un principe de relativité (non totalisateur) et d'une axiomatique dualiste (non réductible) qui fournissent aussitôt les conditions constitutives de l'action collective, si l'on veut qu'elle soit à la fois concevable et observable.

Comme dans toute démarche scientifique, cette axiomatique fondatrice ne reconstitue pas une métaphysique ex ante. Elle ne peut se prévaloir que de sa fécondité explicative et de sa puissance générative. Celles-ci ont été mises à l'épreuve dans de multiples domaines académiques (théorie des mythes rationnels, théorie des apprentissages croisés, théorie des processus d'innovation, théorie des marchés à prescripteurs, histoire des phénomènes de gestion, épistémologie générique etc...) et sur de nombreux terrains d'expérimentation qu'il n'est pas utile de détailler ici, car elles ont fait l'objet de nombreuses publications.

Baptiste Rappin a noté que «l'action » est une catégorie centrale de ces travaux. Mais il ne s'agit pas de poser une ontologie de l'action pour en déduire après coup, et de manière séparée, une dynamique, une politique ou une éthique. Il faut, au contraire, se doter des modèles de l'action collective les plus génériques possibles : ceux qui postulent l'indissociabilité de la dynamique, du politique et de l'éthique. De même qu'en physique il serait peu fécond de décrire la matière soit en termes de forces, soit en termes de géométrie, soit en termes d'énergie. L'énoncé même d'un principe de relativité ou d'une axiomatique de l'action 
conduit à les traiter ensemble, mais sans les rendre indiscernables! Dans cette démarche, l'ontologie ne précède pas nécessairement le modèle théorique, elle peut en découler. Quand une percée théorique advient, elle stimule aussi une reconstruction ontologique.

2. La deuxième critique n'appelle qu'une mise au point. L'auteur voit dans la théorie de l'action collective une nouvelle version de la pensée cybernétique. Or, la cybernétique n'a jamais pu constituer un corpus unifié ; elle a connu de nombreuses tentatives de refondation (on parle couramment de cybernétique de deuxième ou de troisième génération). En outre, les quelques idées centrales du mouvement n'ont guère fait de place à la question des constructions politiques et sociales. De façon prédominante, il s'agissait de rechercher des mécanismes universels de gouvernement, de régulation et de contrôle, indépendamment des relations (humanité, civisme, socialité, droits,...) qui se construisent entre des humains (ou entre des humains et le reste du monde). La cybernétique qui nait au milieu du 20è siècle était une réduction mutilante de l'ancienne "gestion» latine. On sait que les Romains ne pouvaient concevoir les actes de gestion sans une théorie de la responsabilité, de la justice et de la «bienveillance » (comme l'a récemment montré Moira Crété ${ }^{2}$ ).

Baptiste Rappin se trompe donc en affirmant que la théorie de l'action collective se ramène in fine à la cybernétique, et par là même, à une technique de gouvernement. Les sciences de gestion modernes, et la théorie de l'action collective, n'ont cessé de dire le contraire: il n'y a pas de technique de gouvernement qui puisse fonctionner indépendamment d'un ensemble de relations (du sujet à lui-même ou à autrui, de pouvoirs, d'appartenances, de socialités,...). Toutefois, la même théorie invite aussi à penser qu'il n'y a pas de relations humaines, pas de rapports entre gouvernants et gouvernés, sans techniques d'action et de gouvernement.

3. Au-delà de ces réponses, il est utile de préciser quelques implications philosophiques de la théorie de l'action collective.

L'un des principaux apports de la théorie de l'action collective consiste précisément à rejeter les dissociations classiques entre technique et société, entre rationalité et responsabilité, entre justesse et justice etc... La théorie de l'action collective rétablit l'ensemble infini et extensible de ces interdépendances par un axiome qui énonce «l'inséparabilité des savoirs et des relations ». On l'a dit, cet

\footnotetext{
${ }^{2}$ Moira Crété : La «gestion » à l'époque romaine : naissance d'une nouvelle catégorie de l'action collective. 2016 Working paper MinesParostech/PSL UMR I3 9217.
} 
axiome ne se justifie que par sa fécondité théorique et empirique. Il disqualifie en tout cas l'hypothèse d'une cybernétique qui serait indépendante d'une anthropologie politique. Mais il rejette tout autant l'hypothèse d'une anthropologie humaine sans techniques d'action sur elle-même et sur le monde.

Munie de l'axiome d'inséparabilité, la théorie de l'action collective échappe à de nombreuses apories philosophiques et historiques que l'on ne peut toutes décrire ici. On peut l'illustrer simplement: le développement d'une chirurgie moins mutilante, plus respectueuse du corps, ne passe pas par la renonciation à la technicisation du corps : mais par une critique à la fois humaine et technique du bistouri ! Ainsi, le développement de la chirurgie non invasive a nécessité une re-technicisation complète du corps (nouveaux instruments, voies de passages, indications précises..). La théorie permet aussi, dans un autre domaine, de comprendre les effets destructeurs des doctrines « séparatistes » : par exemple, celles des doxa économiques qui pensent l'efficacité économique indépendamment des solidarités sociales; ou à l'inverse, celles des théories politiques qui ignorent les limites des techniques de gouvernement.

Il faut ensuite remarquer que la théorie de l'action collective ne détermine pas une ontologie (et donc une métaphysique) mais une infinité d'ontologies compatibles avec l'axiome de non-séparabilité. Et c'est là un signe profond de cohérence. La théorie n'arrête ni l'histoire ni la puissance créative du vivant. Elle invite ainsi à rejeter toute pensée de l'Etre, de la Nature, ou de la substance qui seraient indépendantes, ou (ce qui revient au même) détermineraient totalement les rapports entre les humains. De même, qu'il serait absurde de penser des relations humaines qui seraient indépendantes des techniques et des connaissances humaines.

Ce n'est que depuis peu d'années que la recherche, toutes disciplines confondues, est confrontée avec ce que l'on appelle ailleurs, un réel nonséparable, des mécanismes de coévolution symbiotiques, ou des dualités existentielles (comme la dualité onde-particule). La théorie de l'action collective participe à ce mouvement. Mais peu de chercheurs sont préparés à ces outils de pensée lorsqu'il s'agit de comprendre l'action collective humaine ou les systèmes incluant des sociétés humaines.

Pour conclure, j'invite donc Baptiste Rappin à poursuivre une réflexion critique plus approfondie sur la théorie de l'action collective et ses implications. Quant à Heidegger, il serait- je l'ai dit- incongru d'engager ici une discussion rigoureuse à son propos. Je me limiterai à indiquer une piste de réflexion pour les lecteurs intéressés par les implications philosophiques de la théorie de l'action collective. Ils pourront aisément vérifier qu'une littérature philosophique abondante atteste 
que le penseur allemand a voulu concevoir une méditation sur l'Etre séparée, et séparable, des questions de responsabilité, de société et de l'idée de commune humanité. Dans les termes de la théorie de l'action collective, ce constat s'exprime clairement: il signifie que cette méditation transgressait dangereusement - l'axiome de non-séparabilité des savoirs et des relations. 